\title{
LOCALLY AFFINE RING EXTENSIONS OF A NOETHERIAN DOMAIN
}

\author{
WILLIAM HEINZER ${ }^{1}$
}

\begin{abstract}
If $A \subset R$ are integral domains with $A$ noetherian, it is shown that if $R$ is contained in an affine ring over $A$ and if for each maximal ideal $P$ of $A$ with $S=A \backslash P, R_{S}$ is an affine ring over $A_{P}$, then $R$ itself is affine over $A$.
\end{abstract}

If $A \subset R$ are (commutative) integral domains and if for each prime ideal $P$ of $A$ with $S=A \backslash P, R_{S}$ is a finitely generated ring extension of $A_{P}$, then we say that $R$ is locally affine over $A$. If for each prime $P$ of $A, R_{S}$ is a polynomial ring over $A_{P}$, then $R$ is said to be locally a polynomial ring over $A$. Even for $A=Z$, the ring of integers, it can happen that $R$ is locally affine over $A$ (and even locally polynomial over $A$ ), but yet $R$ is not finitely generated over $A$. Eakin and Silver in [1] give the following example. Let $\left\{p_{i}\right\}_{i=1}^{\infty}$ be the set of prime integers in $Z$ and let $X$ be an indeterminate. Then $R=Z\left[\left\{X \mid p_{i}\right\}_{i=1}^{\infty}\right]$ is locally a polynomial ring in one variable over $Z$, but $R$ is not finitely generated over $Z$. The question thus naturally arises of what additional conditions on $R$, locally affine over $A$, will imply that $R$ is finitely generated over $A$. Eakin and Silver [1] consider the question of whether $R$ locally a polynomial ring over $A$ and contained in an affine ring over $A$ imply $R$ is finitely generated over $A$. They prove this to be the case when $A$ is a Krull domain, when $A$ is a pseudo-geometric domain, or when $A$ is a 1-dimensional noetherian domain, and raise the question of whether in general $R$ locally polynomial over a noetherian domain $A$ and contained in an affine ring over $A$ imply $R$ is an affine ring over $A$. That the answer is yes is a consequence of the following.

THEOREM. Let $A \subset R$ be integral domains with $A$ noetherian and $R$ locally affine over $A$. If $R$ is contained in an affine ring over $R$, then $R$ itself is affine over $A$.

Received by the editors January 25, 1972.

AMS 1970 subject classifications. Primary 13E05, 13F20, 13 G05.

Key words and phrases. Noetherian integral domain, affine ring, associated prime ideals, localization.

${ }^{1}$ The author received partial support for this research from National Science Foundation grant GP-29326. 
In proving this, our main theorem, we will make use of some properties of the representation of a noetherian integral domain $D$ in the form $D=\bigcap_{\alpha}\left\{D_{P_{\alpha}} \mid P_{\alpha}\right.$ is an associated prime of a principal ideal of $\left.D\right\}$. In general, if $\left\{V_{\alpha}\right\}$ is a collection of integral domains with quotient field $K$, then $\left\{V_{\alpha}\right\}$ is said to have finite character if each nonzero elcment of $K$ is a unit in all but finitely many of the $V_{\alpha}$. If $D=\bigcap_{\alpha} V_{\alpha}$ also has quotient field $K$ and $\left\{V_{\alpha}\right\}$ has finite character, then the representation $D=\bigcap_{\alpha} V_{\alpha}$ is said to be locally finite [3, p. 76]. Note that $L=\bigcap_{\alpha} V_{\alpha}$ is locally finite if and only if each nonzero element of $D$ is a unit in all but finitely many of the $V_{\alpha}$. For an arbitrary integral domain $D$, it is well known that if $\left\{P_{\alpha}\right\}$ is a collection of prime ideals of $D$ such that each associated primc of a principal ideal of $D$ is contained in some $P_{\alpha}$, then $D=\bigcap_{\alpha} D_{P_{\alpha}}$ (see [4, p. 118] or [3, p. 34]). When $D$ is noetherian one has, moreover, the following.

LEMMA 1. If $D$ is a noetherian domain, then the representation $D=$ $\bigcap_{\alpha}\left\{D_{P_{\alpha}} \mid P_{\alpha}\right.$ is an associated prime of a principal ideal of $\left.D\right\}$ is locally finite.

Proof. Since $D$ is noetherian, if $P$ is an associated prime of a principal ideal of $D$, then $P$ is an associated prime of any nonzero $y \in P$. Thus, nonzero elements of $D$ are contained in only finitely many of the $P_{\alpha}$ and the lemma follows.

LEMMA 2. Let $D$ be a noetherian domain, let $\left\{P_{\alpha}\right\}$ be the set of associated primes of principal ideals of $D$, and let $S$ be a multiplicative system in $D$. Then $D_{S}=\bigcap_{\alpha}\left\{D_{P_{\alpha}} \mid S \cap P_{\alpha}=\varnothing\right\}$.

Proof. Each associated prime of a principal ideal in $D_{S}$ is of the form $P_{\alpha} D_{S}$ where $P_{\alpha} \cap S=\varnothing$. Since for $P_{\alpha} \cap S=\varnothing, D_{P_{\alpha}}=\left(D_{S}\right)_{P_{\alpha} D_{S}}$, the result follows.

We note the following immediate consequence of Lemma 2.

Lemma 3. Let $D \subset R$ be integral domains with $D$ noetherian and $R \subset$ $D[1 / f]$ for some nonzero $f \in D$. Let $\left\{P_{\alpha}\right\}$ be the set of associated primes of principal ideals in $D$ and let $P_{1}, \cdots, P_{n}$ be the $P_{\alpha}$ which contain $f$. If $s$ is $a$ nonzero element of $D$ such that $R \notin D_{P_{i}}$ implies $s \in P_{i}$, then $R \subset D[1 / s]$.

We shall also make use of the following standard lemma.

Lemma 4. Let $A$ be a commutative ring with identity and let $R$ be an $A$ algebra. If for each maximal ideal $P$ of $A$ there exists $s \in A \backslash P$ such that $R[1 / s]=R \otimes_{A} A[1 / s]$ is a finitely generated $A[1 / s]$-algebra, then $R$ is $a$ finitely generated A-algebra.

Proof. Let $\left\{P_{\beta}\right\}$ be the set of maximal ideals of $A$ and let $s_{\beta} \in A \backslash P_{\beta}$ be such that $R\left[1 / s_{\beta}\right]$ is a finitely generated $A\left[1 / s_{\beta}\right]$-algebra. Then $\left(\left\{s_{\beta}\right\}\right)=A$, so there exist $s_{1}, \cdots, s_{n} \in\left\{s_{\beta}\right\}$ such that $\left(s_{1}, \cdots, s_{n}\right)=A$. If $N_{i}$ is a finite 
subset of $R$ such that $R\left[1 / s_{i}\right]=A\left[1 / s_{i}, N_{i}\right]$, then $N=N_{1} \cup \cdots \cup N_{n}$ is such that $R=A[N]$.

Proof of the Theorem. Let $P$ be a maximal ideal of $A$, let $S=A \backslash P$, and let $x_{1}, \cdots, x_{n} \in R$ be such that $A_{S}\left[x_{1}, \cdots, x_{n}\right]=R_{S}$. By Lemma 4, it will suffice to show for some $s \in S$ that $R[1 / s]=A\left[1 / s, x_{1}, \cdots, x_{n}\right]$. Let $D=A\left[x_{1}, \cdots, x_{n}\right]$. Then $D \subset R$ and $R$ is contained in the quotient field of $D$. By assumption, $R$ is contained in an affine ring over $A$, so we have $R \subset D\left[\xi_{1}, \cdots, \xi_{m}\right]$. By taking a residue class ring of $D\left[\xi_{1}, \cdots, \xi_{m}\right]$ modulo a prime ideal lying over $(0)$ in $R$, we may assume that $D\left[\xi_{1}, \cdots, \xi_{m}\right]$ is an integral domain. Applying the normalization lemma of $[4$, p. 45], we may reduce modulo a suitable prime ideal to the case where the $\xi_{i}$ are algebraic over $D$. Finally, writing the $\xi_{i}$ with denominators in $D$ and using the fact that $R$ is contained in the quotient field of $D$, we get a nonzero $f \in D$ such that $R \subset D[1 / f]$. (A reduction to this case is also given by Eakin and Silver [1].) Since $A$ is noetherian, $D=A\left[x_{1}, \cdots, x_{n}\right]$ is noetherian. Let $\left\{P_{\alpha}\right\}$ be the set of associated primes of principal ideals in $D$. Note that if $f \notin P_{\alpha}$, then $D[1 / f] \subset D_{P_{\alpha}}$, so $R \subset D_{P_{\alpha}}$. Let $P_{1}, \cdots, P_{m}$ be the $P_{\alpha}$ which contain $f$. If $R \notin D_{P_{i}}$, then $P_{i} \cap A \notin P$; for $R \subset A_{S}\left[x_{1}, \cdots, x_{n}\right]$ and $P_{i} \cap A \subset P$ implies $A_{S}\left[x_{1}, \cdots, x_{n}\right] \subset D_{P_{i}}$. Hence we can choose $s \in A \backslash P$ such that $R \notin D_{P_{i}}$ implies $s \in P_{i}$. By Lemma 3, $R \subset D[1 / s]=$ $A\left[1 / s, x_{1}, \cdots, x_{n}\right]$, which completes the proof of the Theorem.

REMARK. It would be interesting to have more general conditions on $A$ and $R$ in order that $R$ locally affine over $A$ implies that $R$ is finitely generated over $A$. In our proof, the fact that $A$ is noetherian was used to insure that if $D$ is a domain finitely generated over $A$ and if $\left\{P_{\alpha}\right\}$ is the set of associated primes of principal ideals of $D$, then the representation $D=\bigcap_{\alpha} D_{P_{\alpha}}$ is locally finite and for any nonzero $s \in D, D[1 / s]=$ $\bigcap_{\alpha}\left\{D_{P_{\alpha}} \mid s \notin P_{\alpha}\right\}$. Perhaps these properties hold for $A$ in a larger class of integral domains, thus allowing generalization of the above theorem by weakening the noetherian hypothesis on $A .^{2}$ It is not true in general, however, for integral domains $A \subset R$ with $R$ locally affine over $A$ and contained in an affine ring over $A$ that necessarily $R$ is finitely generated over $A$. The following example illustrates this.

EXAMPLE. Let $A^{*} \subset R^{*}$ be integral domains with $R^{*}$ locally affine over $A^{*}$, but $R^{*}$ not finitely generated over $A^{*}$ (e.g. we could take the example of Eakin and Silver mentioned above, $A^{*}=Z$ and $\left.R^{*}=Z\left[\left\{X \mid p_{i}\right\}_{i=1}^{\infty}\right]\right)$. Let $K$ be the quotient field of $R^{*}$ and let $M$ be the maximal ideal of the formal

${ }^{2}$ If $R$ is locally a polynomial ring over $A$, then it is sufficient to have these properties for $D$ a polynomial ring over $A$. Thus, for example, as Eakin and Silver show in [1] , if $A$ is a Krull domain, then $R$ locally polynomial over $A$ and contained in an affine ring over $A$ imply $R$ is affine over $A$. This result easily generalizes to the case where $A$ has a locally finite representation, $A=\bigcap_{\beta} A_{P^{\prime} \beta}$, with the $A_{P^{\prime} \beta}$ rank one valuation rings. 
power series ring $K[[Y]]$. Let $A=A^{*}+M$ and $R=R^{*}+M$. Then $M$ is a common prime ideal of $A$ and $R$ and $M$ compares with any other prime ideal of $A$ or $R$ (see for example [2, p. 560]). It is now easily verified that $R$ is locally affine over $A$ and $R \subset A[1 / Y]$, so $R$ is contained in an affine ring over $A$. But if $R$ were finitely generated over $A$, then $R^{*}=R / M$ would be finitely generated over $A^{*}=A / M$ which it is not.

\section{REFERENCES}

1. P. Eakin and J. Silver, Rings which are almost polynomial rings, Trans. Amer. Math. Soc. (to appear).

2. R. W. Gilmer, Multiplicative ideal theory, Queen's Papers in Pure and Appl. Math., no. 12, Queen's University, Kingston, Ont., 1968. MR 37 \#5198.

3. I. Kaplansky, Commutative rings, Allyn and Bacon, Boston, Mass., 1970. MR 40 $\# 7234$.

4. M. Nagata, Local rings, Interscience Tracts in Pure and Appl. Math., no. 13, Interscience, New York, 1962. MR 27 \#5790.

Department of Mathematics, Purdue University, Lafayette, Indiana 47907 